\title{
Analysis and experiments of adaptive feedforward and combined vibration control system with variable step size and reference filter
}

\author{
Lingbo Xie, Zhi-cheng Qiu*, Xian-min Zhang \\ School of Mechanical and Automotive Engineering, South China University of Technology, Guangzhou 510641, PR China
}

\begin{abstract}
An adaptive feedforward and combined vibration control system with variable step size and reference filter is analyzed and implemented to suppress the vibration of a kind of thin-walled structure. The theoretical analysis and experiments of adaptive feedforward and combined vibration control algorithms are presented. The experimental setup of a flexible plate bonded PZT (Lead Zirconate Titanate) sensors/actuators is constructed. The control algorithm combines an adaptive feedforward controller and an adaptive feedback controller together, which has shown a superior control performance in the experiments. Considering the disadvantages of fixed step size used in the conventional filtered-U least mean square (FULMS) control algorithm, a variable step-size (VSS) method is applied. Also, a reference filter is used to extract the desired signal from the positive feedback and measurement noise. A proportional derivative (PD) feedback control algorithm is also applied as comparison. The experimental results demonstrate that the adaptive combined control algorithm has better control performance than the adaptive feedforward and PD feedback control algorithms.
\end{abstract}

Keywords Adaptive feedforward, Adaptive combined, Variable step size, Reference filter

\section{Introduction}

Thin-walled structures have been widely used in aerospace structures and industrial applications with advantages of low weight and low energy consumption [1]. The design of thin-walled space structures trends toward large size, high flexibility and low stiffness. Thus, the vibration or flutter can be easily excited by external disturbances [2]. The serious vibration can even result in the destructive damage of the thin-walled structures without effective control [1]. Therefore, it is necessary to investigate active vibration control algorithms to suppress the vibration of the thin-walled structures. Now, piezoelectric materials have been widely utilized in the form of piezoelectric patches as both actuators and sensors for vibration control of flexible plates. Osama Abdeljaber et al. [3] introduced a new intelligent methodology to mitigate the vibration response of flexible cantilever plates by using the piezoelectric sensor /actuator pairs to control the vibration of plates. Sadek et al. [4] used piezoelectric patch actuators for active control of simply supported flexible plates.

The adaptive feedforward control algorithms for active vibration and noise control have developed very fast in

\footnotetext{
* Corresponding author. Tel.: +86-20-8711 $4635(\mathrm{O})$.

E-mail address: zhchqiu@ scut.edu.cn; zhchqiu@126.com (Zhi-cheng Qiu)
} 
recent years. Jinhao Qiu and Masakazu Haraguchi [5] employed piezoelectric patches along with a controller that utilizes finite impulse response filter and the filtered-X LMS algorithm to control the response of flexible plates. FULMS algorithm was proposed firstly for active noise control [6]. Ho-Wuk Kim et al. [7] used a kind of modified FULMS algorithm for active noise control and the experimental results demonstrated that it can be effectively applicable to a short acoustic duct. A novel adaptive algorithm with an IIR filter and a variable step size was presented for active noise control in a short duct, which has a superior convergence performance compared with the conventional algorithm [8]. Zhiyuan Gao et al. [9] used the MIMO FULMS vibration controller to control the configured smart beam; the control algorithm has a good control performance to suppress the vibration significantly with rapid convergence. When we try to combine the merits of the conventional filtered-U least mean square (FULMS) control algorithm and variable step size (VSS) together, the FUVSSLMS can be developed and used as the abbreviation of the new control algorithm. An improved FULMS algorithm with variable step size was used to control the resonant vibration of a flexible clamped-clamped plate with laser displacement sensor measurement effectively [10]. Khaled Mayyas [11] proposed a variable step-size selective partial update least mean square (LMS) algorithm; and experiments indicated that the proposed algorithm performed as well as other algorithms while requiring less computational complexity. Generally speaking, the variable step-size control algorithms with a fast convergence speed and low residual error have shown better control performance than the fixed step-size control algorithms.

However, the control performance of the adaptive feedforward control algorithm will be seriously weakened by the positive feedback between the control part and reference sensor. The positive feedback exists in an active vibration control system and it may result in system instability and system crashes [12]. The modified positive position feedback (MPPF) controller using collocated piezoelectric actuators and sensors is developed as an adaptive controller to provide significant vibration reduction on a cantilever beam [13]. Different from some control algorithms in which the reference signal is directly extracted from the signal generator, the reference signal extracted from the reference sensor is widely used in practice. It means that the reference signal will be influenced by the positive feedback and measurement noise. Alexander D. Streeter [14] presented a kind of architecture for both stationary and non-stationary noise control by combining feedforward and feedback control. An adaptive feedforward controller based on the filtered-U recursive least square (FURLS) algorithm was proposed to remove the impacts of positive feedback [15]. Ioan Dor'e Landau et al. [16] provided a general adaptive feedforward compensation algorithm for active vibration control with mechanical coupling and local feedback, which had been applied to an active vibration control system. In order to solve the problem of structural positive feedback, a hybrid adaptive vibration control algorithm by combining an adaptive feedforward controller and an adaptive feedback controller was proposed and the experimental results confirmed the feasibility and effectiveness of the proposed algorithm [17]. Shouda Jiang et al. [18] proposed a new hybrid NANC system capable of reducing the primary noise and the uncorrelated disturbance simultaneously. S.R. Chen et al. [19] adopted a combined 
adaptive algorithm with two low pass filters to explore the effects of sound elimination in a cylindrical duct, and the experiment showed that the effect of noise reduction in ANC system was influenced extremely by the reference signal. In conclusion, the conventional adaptive feedforward algorithm can not solve the issue of positive feedback completely and the reference signal measured by reference sensor should be preprocessed before it is calculated into the control algorithm. Then it is clear that by using a reference filter one can design an adaptive combined FUVSSLMS control algorithm including an adaptive feedforward controller and an adaptive feedback controller to actively control the vibration and noise.

This paper investigates a kind of adaptive feedforward and combined vibration control system with variable step size and a reference filter. The active vibration control experimental setup with PZT sensors and PZT actuators is constructed. The theoretical analysis and experiments of adaptive feedforward and combined vibration control algorithm with variable step size and reference filter are carried out. A proportional derivative (PD) feedback control algorithm is also used in the experiments as a comparison. The experimental results demonstrate that the adaptive combined FUVSSLMS control algorithm has better control performance than the other two control algorithms.

\section{Controller Design}

\subsection{The adaptive feedforward FUVSSLMS control algorithm with variable step size and reference filter}

As mentioned before, the FUVSSLMS control algorithm can be defined as the combination of the conventional filtered-U least mean square (FULMS) with variable step size (VSS). Then the block diagram of the adaptive feedforward FUVSSLMS control algorithm with variable step size and reference filter is shown in Figure 1 [10, 20].

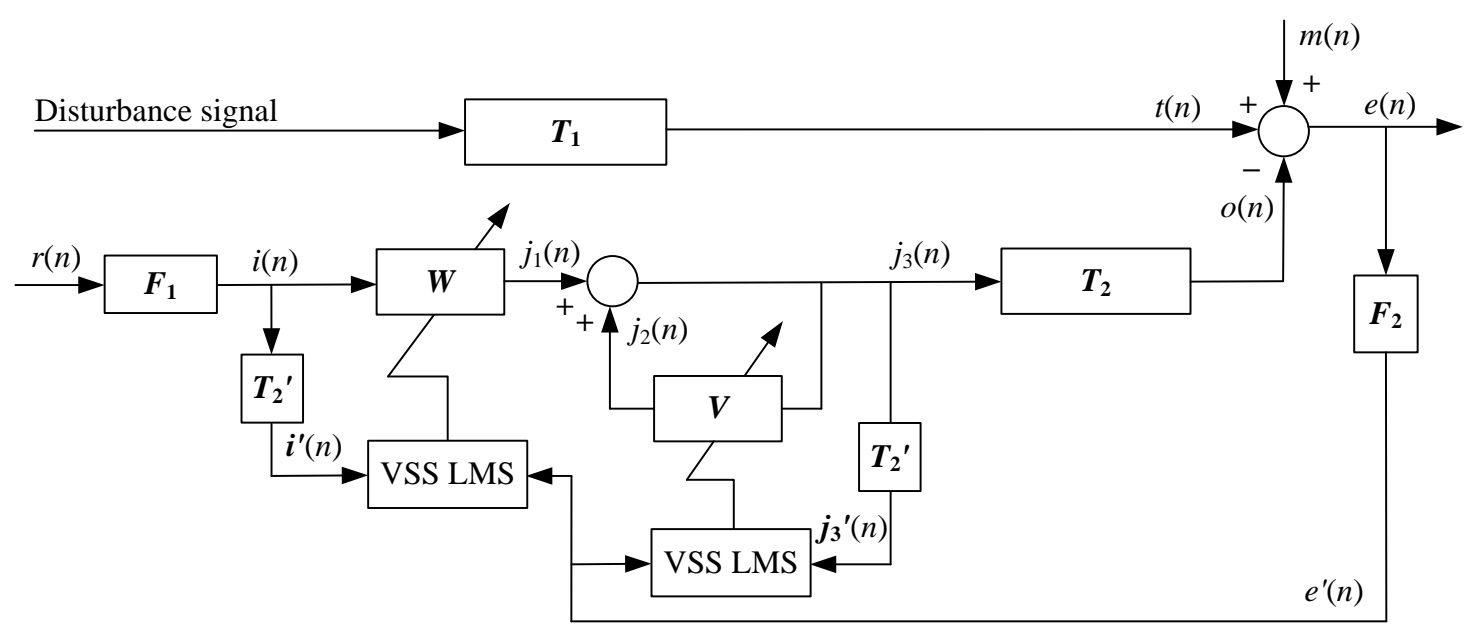

Figure 1. Scheme of the adaptive feedforward FUVSSLMS control algorithm with variable step size and reference filter. 
Different from other conventional FULMS control algorithms, the reference signal $r(n)$ is not directly extracted from the signal generator. It is extracted from the reference sensor, which means $r(n)$ will be influenced by the positive feedback and measurement noise inevitably. Then a reference filter $\boldsymbol{F}_{1}$ is designed and used to extract the desired signal from the positive feedback and measurement noise, and the filtering effect will be shown in the following experiments.

In fact, it is inevitable that the error signal $e(n)$ will contain some uncorrelated measurement noise $m(n)$ which will affect the control performance. Similar to $\boldsymbol{F}_{1}$, a error filter $\boldsymbol{F}_{2}$ is used to obtain the desired signal $e^{\prime}(n)$.

The iterative process of the adaptive feedforward FUVSSLMS control algorithm can be summarized as follows $[6,10,20]$

$$
\left\{\begin{array}{l}
j_{3}(n)=j_{1}(n)+j_{2}(n)=\boldsymbol{I}(n) \boldsymbol{W}^{\mathrm{T}}(n)+\boldsymbol{J}_{3}(n-1) \boldsymbol{V}^{\mathrm{T}}(n) \\
e(n)=t(n)+m(n)-o(n) \\
\boldsymbol{W}(n+1)=\boldsymbol{W}(n)+2 \alpha(n) e^{\prime}(n) \boldsymbol{i}^{\prime}(n) \\
\boldsymbol{V}(n+1)=\boldsymbol{V}(n)+2 \beta(n) e^{\prime}(n) \boldsymbol{j}_{3}^{\prime}(n-1)
\end{array},\right.
$$

where

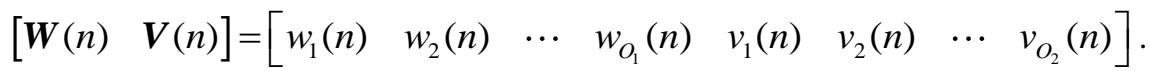

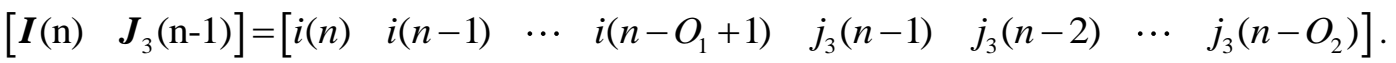

$$
\begin{aligned}
& {\left[\boldsymbol{i}^{\prime}(n)\right]^{T}=\left[\begin{array}{cccc}
i(n) & i(n-1) & \cdots & i(n-d+1) \\
i(n-1) & i(n-2) & \cdots & i(n-d) \\
\vdots & \vdots & \vdots & \vdots \\
i\left(n-O_{1}+1\right) & i\left(n-O_{1}\right) & \cdots & i\left(n-O_{1}-d+2\right)
\end{array}\right]\left[\begin{array}{c}
t_{1} \\
t_{2} \\
\vdots \\
t_{d}
\end{array}\right]=\boldsymbol{I}_{1}(n) \boldsymbol{T}_{2}^{\prime}} \\
& {\left[\boldsymbol{j}_{3}^{\prime}(n-1)\right]^{T}=\left[\begin{array}{cccc}
j_{3}(n-1) & j_{3}(n-2) & \cdots & j_{3}(n-d) \\
j_{3}(n-2) & j_{3}(n-3) & \cdots & j_{3}(n-d-1) \\
\vdots & \vdots & \vdots & \vdots \\
j_{3}\left(n-O_{2}\right) & j_{3}\left(n-O_{2}-1\right) & \cdots & j_{3}\left(n-O_{2}-d+1\right)
\end{array}\right]\left[\begin{array}{c}
t_{1} \\
t_{2} \\
\vdots \\
t_{d}
\end{array}\right]=\boldsymbol{J}_{1}(n-1) \boldsymbol{T}_{2}{ }^{\prime}} \\
& e(n)=t(n)+m(n)-o(n)=t(n)+m(n)-\left(\boldsymbol{W}(n) \boldsymbol{I}_{1}(n)+\boldsymbol{V}(n) \boldsymbol{J}_{1}(n-1)\right) \boldsymbol{T}_{2}^{\prime} \\
& =t(n)+m(n)-\boldsymbol{W}(n)\left(\boldsymbol{I}_{1}(n) \boldsymbol{T}_{2}^{\prime}\right)-\boldsymbol{V}(n)\left(\boldsymbol{J}_{1}(n-1) \boldsymbol{T}_{2}^{\prime}\right) \\
& =t(n)+m(n)-\boldsymbol{W}(n)\left[\boldsymbol{i}^{\prime}(n)\right]^{T}-\boldsymbol{V}(n)\left[\boldsymbol{j}_{3}{ }^{\prime}(n-1)\right]^{T}
\end{aligned}
$$

Since the theory of the FULMS algorithm has been explained in references [6] and [20] in detail, one can ignore the explanation and focus on the main points to make the paper concise. The meaning of the given symbols is shown in Table 1. The symbols of the adaptive combined FUVSSLMS control algorithm are also included. 
Table 1 . The meaning of the given symbols.

\begin{tabular}{|c|c|}
\hline Symbol & Meaning \\
\hline$n$ & $n$th sampling instant \\
\hline$r(n)$ & Reference signal \\
\hline$i(n)$ & Filtered signals from $r(n)$ \\
\hline$t(n)$ & Disturbance signal response of the controlled structure \\
\hline$e(n)$ & Error signal \\
\hline$o(n)$ & Control response of the controlled structure \\
\hline$m(n)$ & Measurement noise \\
\hline$j(n)$ & Control output of the adaptive filter \\
\hline$j_{1}(n)$ & Output of the feedforward filter $\boldsymbol{W}$ \\
\hline$j_{2}(n)$ & Output of the feedback filter $\boldsymbol{V}$ \\
\hline$j_{3}(n)$ & Sum of $j_{1}(n)$ and $j_{2}(n)$ \\
\hline$j_{4}(n)$ & Output of the feed-forward filter $\boldsymbol{W}_{1}$ \\
\hline$j_{5}(n)$ & Output of the feedback filter $V_{1}$ \\
\hline$j_{6}(n)$ & Sum of $j_{4}(n)$ and $j_{5}(n)$ \\
\hline$j^{\prime}(n)$ & Filtered signals from $j(n)$ \\
\hline$e^{\prime}(n)$ & Filtered signals from $e(n)$ \\
\hline$i^{\prime}(n)$ & Filtered signals from $i(n)$ \\
\hline$j_{3}^{\prime}(n)$ & Filtered signals from $j_{3}(n)$ \\
\hline $\boldsymbol{h}^{\prime}(n)$ & Filtered signals from $h(n)$ \\
\hline$j_{6}^{\prime}(n)$ & Filtered signals from $j_{6}(n)$ \\
\hline $\boldsymbol{F}_{1}$ & Reference filter \\
\hline $\boldsymbol{F}_{2}$ & Error filter \\
\hline $\boldsymbol{T}_{1}$ & Primary path from the disturbance signal to the error sensor \\
\hline $\boldsymbol{T}_{2}$ & Control path from the controlling part to the error sensor \\
\hline$T_{2}^{\prime}$ & Estimation of $\boldsymbol{T}_{2}$ \\
\hline $\boldsymbol{W}$ & Adaptive feedforward controller \\
\hline $\boldsymbol{V}$ & Adaptive feedback controller \\
\hline $\boldsymbol{W}_{1}$ & Adaptive feedforward controller \\
\hline$V_{1}$ & Adaptive feedback controller \\
\hline$O_{1}$ & Order of $\boldsymbol{W}$ \\
\hline $\mathrm{O}_{2}$ & Order of $\boldsymbol{V}$ \\
\hline $\mathrm{O}_{3}$ & Order of $\boldsymbol{W}_{1}$ \\
\hline $\mathrm{O}_{4}$ & Order of $\boldsymbol{V}_{1}$ \\
\hline$\alpha$ & Convergence factor of $\boldsymbol{W}$ \\
\hline$\beta$ & Convergence factor of $\boldsymbol{V}$ \\
\hline$\gamma$ & Convergence factor of $\boldsymbol{W}_{1}$ \\
\hline$\varphi$ & Convergence factor $V_{1}$ \\
\hline$d$ & Order of $\boldsymbol{T}_{2}^{\prime}$ \\
\hline VSS LMS & Variable step size least mean square \\
\hline
\end{tabular}




\subsection{The adaptive combined FUVSSLMS control algorithm with variable step size and reference filter}

The block diagram of the adaptive combined FUVSSLMS control algorithm is shown in Figure 2. From Figure 2, it can be seen that there are two main improvements, i.e., denoted in blue box compared with the adaptive feedforward FUVSSLMS control algorithm. The adaptive combined FUVSSLMS control algorithm is composed of an adaptive feedforward controller and an adaptive feedback controller to control the vibration. The meaning of the given symbols has also been shown in Table 1. From Figure 2, it can be seen that the sum of the control output can be expressed as

$$
j(n)=j_{3}(n)+j_{6}(n) .
$$

In order to obtain the reference signal $h(n)$ of the adaptive feedback controller, the reference signal extraction technology is employed as $[10,21]$

$$
h(n)=j^{\prime}(n)+e^{\prime}(n-1)=\boldsymbol{J}(n-1) \boldsymbol{T}_{2}^{\prime}+e^{\prime}(n-1),
$$

where

$$
\boldsymbol{J}(n-1)=\left[\begin{array}{llll}
j(n-1) & j(n-2) & \cdots & j(n-d)
\end{array}\right]
$$

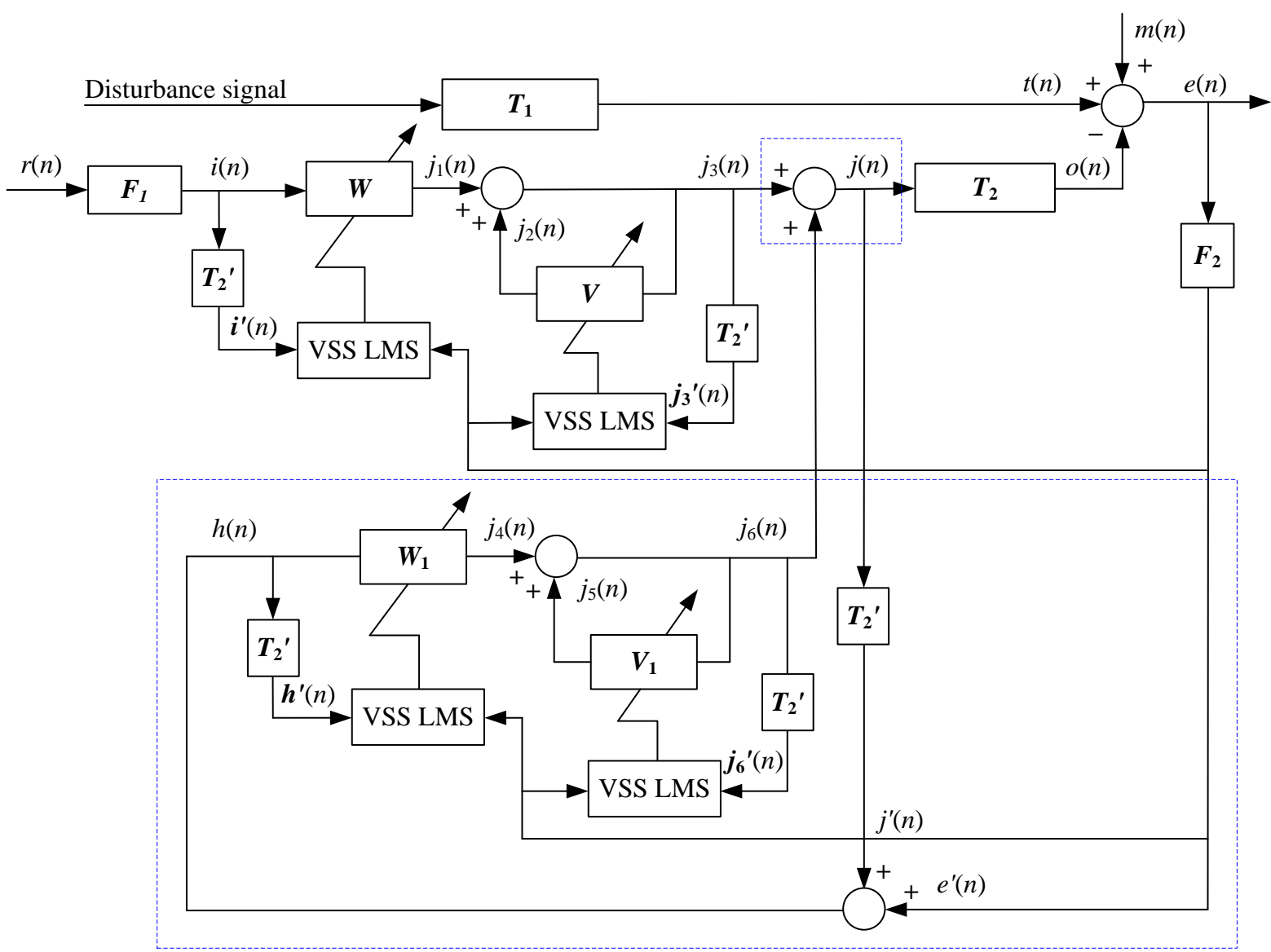

Figure 2. Scheme block diagram of the adaptive combined FUVSSLMS control algorithm with variable step size and reference filter. 
In Figure 2, it can be seen that nearly all the equations of the adaptive feedforward controller are the same as those of the adaptive feedforward FUVSSLMS control algorithm shown in Figure 1; except the expression of $e(n)$ which is also used for the adaptive feedback controller as the common error. Then it is only needed to demonstrate the equations of the adaptive feedback controller as follows

$$
\left\{\begin{array}{l}
j_{6}(n)=j_{4}(n)+j_{5}(n)=\boldsymbol{H}(n) \boldsymbol{W}_{1}^{\mathrm{T}}(n)+\boldsymbol{J}_{6}(n-1) \boldsymbol{V}_{1}^{\mathrm{T}}(n) \\
e(n)=t(n)+m(n)-o(n) \\
\boldsymbol{W}_{1}(n+1)=\boldsymbol{W}_{1}(n)+2 \gamma(n) e^{\prime}(n) \boldsymbol{h}^{\prime}(n) \\
\boldsymbol{V}_{1}(n+1)=\boldsymbol{V}_{1}(n)+2 \varphi(n) e^{\prime}(n) \boldsymbol{j}_{6}^{\prime}(n-1)
\end{array},\right.
$$

where

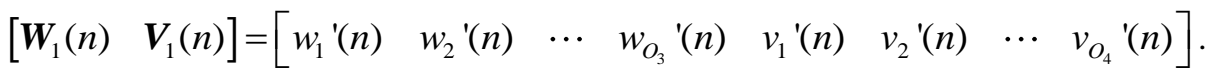

$$
\begin{aligned}
& {\left[\begin{array}{ll}
\boldsymbol{H}(\mathrm{n}) & \boldsymbol{J}_{6}(\mathrm{n}-1)
\end{array}\right]=\left[\begin{array}{llllllll}
h(n) & h(n-1) & \cdots & h\left(n-O_{3}+1\right) & j_{6}(n-1) & j_{6}(n-2) & \cdots & j_{6}\left(n-O_{4}\right)
\end{array}\right] .}
\end{aligned}
$$

$$
\left[\boldsymbol{h}^{\prime}(n)\right]^{T}=\left[\begin{array}{cccc}
h(n) & h(n-1) & \cdots & h(n-d+1) \\
h(n-1) & h(n-2) & \cdots & h(n-d) \\
\vdots & \vdots & \vdots & \vdots \\
h\left(n-O_{3}+1\right) & h\left(n-O_{3}\right) & \cdots & h\left(n-O_{3}-d+2\right)
\end{array}\right]\left[\begin{array}{c}
t_{1} \\
t_{2} \\
\vdots \\
t_{d}
\end{array}\right]=\boldsymbol{H}_{1}(n) \boldsymbol{T}_{2}^{\prime}
$$$$
\left[\boldsymbol{j}_{6}^{\prime}(n-1)\right]^{T}=\left[\begin{array}{cccc}
j_{6}(n-1) & j_{6}(n-2) & \cdots & j_{6}(n-d) \\
j_{6}(n-2) & j_{6}(n-3) & \cdots & j_{6}(n-d-1) \\
\vdots & \vdots & \vdots & \vdots \\
j_{6}\left(n-O_{4}\right) & j_{6}\left(n-O_{4}-1\right) & \cdots & j_{6}\left(n-O_{4}-d+1\right)
\end{array}\right]\left[\begin{array}{c}
t_{1} \\
t_{2} \\
\vdots \\
t_{d}
\end{array}\right]=\boldsymbol{J}_{1}^{\prime}(n-1) \boldsymbol{T}_{2}^{\prime} .
$$

$$
\begin{aligned}
e(n) & =t(n)+m(n)-o(n) \\
& =t(n)+m(n)-\left(\boldsymbol{W}_{1}(n) \boldsymbol{H}_{1}(n)+\boldsymbol{V}_{1}(n) \boldsymbol{J}_{1}^{\prime}(n-1)+\boldsymbol{W}(n) \boldsymbol{I}_{1}(n)+\boldsymbol{V}(n) \boldsymbol{J}_{1}(n-1)\right) \boldsymbol{T}_{2}^{\prime} \\
& =t(n)+m(n)-\boldsymbol{W}_{1}(n)\left(\boldsymbol{H}_{1}(n) \boldsymbol{T}_{2}^{\prime}\right)-\boldsymbol{V}_{1}(n)\left(\boldsymbol{J}_{1}^{\prime}(n-1) \boldsymbol{T}_{2}^{\prime}\right)-\boldsymbol{W}_{\boldsymbol{1}}(n)\left(\boldsymbol{I}_{1}(n) \boldsymbol{T}_{2}^{\prime}\right)-\boldsymbol{V}_{1}(n)\left(\boldsymbol{J}_{1}(n-1) \boldsymbol{T}_{2}^{\prime}\right) \\
& =t(n)+m(n)-\boldsymbol{W}_{1}(n)\left[\boldsymbol{h}^{\prime}(n)\right]^{T}-\boldsymbol{V}_{1}(n)\left[\boldsymbol{j}_{6}^{\prime}(n-1)\right]^{T}-\boldsymbol{W}_{1}(n)\left[\boldsymbol{i}^{\prime}(n)\right]^{T}-\boldsymbol{V}_{1}(n)\left[\boldsymbol{j}_{3}^{\prime}(n-1)\right]^{T}
\end{aligned}
$$

On one hand, from the above equations one can see that the value of $e(n)$ in equation (15) is smaller than that in equation (6), which means that the control performance of the adaptive combined FUVSSLMS control algorithm will be better than that of the adaptive feedforward FUVSSLMS control algorithm theoretically. On the other hand, it can be seen that the convergence rate of the adaptive feedforward and feedback filters is proportional to the value of $e^{\prime}(n)$ from equation (1) and (10), which means that it may take more time for the adaptive combined FUVSSLMS control algorithm to accomplish the adaptive processing than the adaptive feedforward FUVSSLMS control algorithm theoretically, but this shortcoming can be eliminated by just using an ordinary computer in the following experiments. 


\subsection{The variable step size (VSS) LMS algorithm based on the sine function}

As previously stated, it is necessary to investigate a kind of simple variable step size when using the adaptive feedforward and adaptive combined FUVSSLMS control algorithms. Due to the understandable theory and simple computation, the sine function is selected as the variable step size. Then the sine function can be chosen as

$$
y=\sin (|x|-\pi / 2)+1 .
$$

Here, $e(n)$ is used to replace $x$ and $\alpha(n)$ is used to replace $y$. Then, one can obtain the variable step size as $[10,22]$

$$
\alpha(n)=a *[\sin (b *|e(n)|-\pi / 2)+1],
$$

where $a$ determines the step-size curve range and the convergence rate; $b$ determines the shape of the step-size curve when it is close to zero.

The bounds of the variable step size $\alpha(n)$ is $0<\alpha(n)<1 / \alpha_{\max } . \alpha_{\max }$ is the maximum eigenvalue of the input signal autocorrelation matrix.

As mentioned above, $\alpha$ is the convergence factor of the feedforward controller $\boldsymbol{W} ; \beta$ is the convergence factor of the feedback controller $\boldsymbol{V} ; \gamma$ is the convergence factor of the feedforward controller $\boldsymbol{W}_{1}$, and $\varphi$ is the convergence factor of the feedback controller $\boldsymbol{V}_{1}$. In order to decrease computer capacity, $\beta(n), \gamma(n)$ and $\varphi(n)$ can be set proportional to $\alpha(n)$ respectively as

$$
\left\{\begin{array}{l}
\beta(n)=\mathrm{c}_{1} \times \alpha(n) \\
\gamma(n)=\mathrm{c}_{2} \times \alpha(n), \\
\varphi(n)=\mathrm{c}_{3} \times \alpha(n)
\end{array}\right.
$$

where $c_{1}, c_{2}, c_{3}$ is the proportional coefficients.

\subsection{Adaptive control path identification}

From Figure 1 and 2, it can be seen that the control path from the control part to the error sensor $\boldsymbol{T}_{2}$ is needed to be identified for using the adaptive feedforward FUVSSLMS control algorithm and the adaptive combined FUVSSLMS control algorithm. Generally speaking, two kinds of system identification methods includes online modeling [21] and offline modeling $[10,20]$ methods. The online modeling method can identify the system in real time at the expense of increasing computation. However, if the system can be assumed generally stable during the control period, then the offline modeling method can be used to identify the control path by using a specific algorithm. In fact, the experimental setup is mainly made up of epoxy resin which is physically stable at normal operating temperature during the control period. Therefore, the offline modeling method can be applied and the identified model $\boldsymbol{T}_{2}{ }^{\prime}$ used in experiments. 


\section{Experiments}

\subsection{Introduction of the experimental setup}

The active vibration control experimental setup of a thin-walled plate regarded as the thin-walled structure is shown in Figure 3. The experimental setup is composed of four parts: a thin-walled plate, the vibration excitation system, the vibration measurement system and the vibration control system. The thin-walled plate is made up of epoxy resin with dimension of $650 \mathrm{~mm} \times 500 \mathrm{~mm} \times 2 \mathrm{~mm}$. PZT actuators for excitation and control are pasted on both surfaces of the thin-walled plate with dimension of $50 \mathrm{~mm} \times 15 \mathrm{~mm} \times 1 \mathrm{~mm}$. The reference PZT sensor and error PZT sensor are bonded only on the top surface of the thin-walled plate with dimension of $40 \mathrm{~mm} \times 10 \mathrm{~mm} \times 1 \mathrm{~mm}$.

Since the location optimization of PZT sensors/actuators has been shown in reference [23], we had better omit the explanation to make the paper concise. In fact, the PZT actuators for excitation/control have been located symmetrically to the middle line, and the reference /error PZT sensor has been located just on the middle line as shown in Figure 3. The material properties of the PZT sensors and actuators are the same, as shown in Table 2. Since the vibration amplitude of the first mode is far greater than that of other modes, only the first mode of vibration is excited and controlled in the experiments. By using the simulation software ANSYS, it can be seen that the first mode vibration conditions of different-sized thin-walled plates are almost the same, which means that all of the maximum vibration amplitudes of the three different-sized plates happen in the center area of the plates, and all of the minimum vibration amplitudes of the three different-sized plates happen in the fixed ends of the plates. Then it is obvious that the proposed algorithm is also applicable for more flexible plates with much lower eigen-frequency. Therefore, in order to reduce the size of experimental setup, one can select the dimension of $650 \mathrm{~mm} \times 500 \mathrm{~mm} \times 2 \mathrm{~mm}$ as an example to illustrate the control performance.

In Figure 4, the schematic diagram of the adaptive feedforward and adaptive combined FUVSSLMS vibration control system is depicted clearly. The operating process of the experimental setup is described as follows: The PZT voltage amplifier I is used to amplify and transmit the first mode excitation signal generated by the signal generator. The PZT actuators for excitation receive the amplified voltage to excite the vibration at the left end of the thin-walled plate. Then the reference PZT sensor and error PZT sensor can measure the analog vibration signals and send them into the data acquisition which can convert analog signals to digital signals. Then, the digital signals are sent to the computer for control algorithm calculation. The digital filter is used to extract the desired signals from the given digital signals. After filtering, the computer will run the control algorithm and send control digital signals to the D/A converter which can convert digital signals to analog signals. Then, these analog signals will be sent to the PZT voltage amplifier II . The amplified control analog signals are applied to the PZT actuators to suppress the vibration. 
Table 2. Material properties of PZT sensors and actuators

\begin{tabular}{ccc}
\hline Property & Unit & Piezoelectric patch \\
\hline Young's modulus & $\mathrm{GPa}$ & 63 \\
Density & $\mathrm{kg} / \mathrm{m} 3$ & 7650 \\
Poisson ratio & - & 0.3 \\
Piezoelectric strain constant & $\mathrm{m} / \mathrm{V}$ & $-166 \times 10-12$ \\
\hline
\end{tabular}

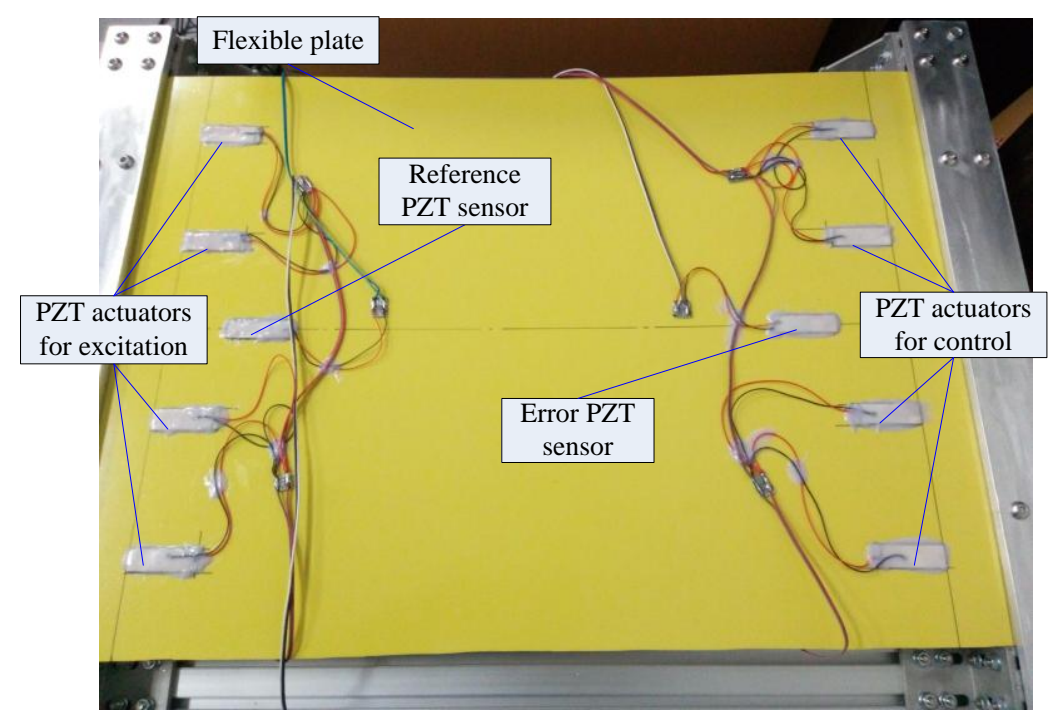

Figure 3. Photograph of the active vibration control experimental setup.

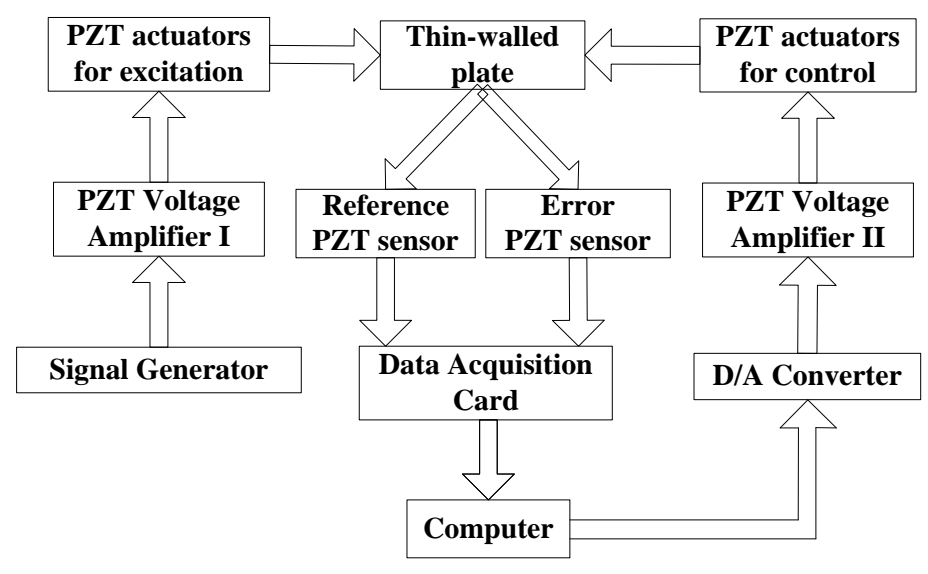

Figure 4. Schematic diagram of the adaptive feedforward and adaptive combined FUVSSLMS vibration control system.

The PZT voltage amplifier I is used to amplify the excitation signal generated by the signal generator to excite the plate, which amplifies the analog voltage ranging from $-5 \mathrm{~V}$ to $+5 \mathrm{~V}$ to a high voltage ranging from $-130 \mathrm{~V}$ to $+130 \mathrm{~V}$. Similarly, the PZT voltage amplifier II can amplify the control analog voltage ranging from $-5 \mathrm{~V}$ to $+5 \mathrm{~V}$ to a high voltage ranging from $-260 \mathrm{~V}$ to $+260 \mathrm{~V}$. The analog signals measured by the reference PZT sensor and error PZT sensor 
are amplified by two charger amplifiers (YE5850) to the analog voltage ranging from $-10 \mathrm{~V}$ to $+10 \mathrm{~V}$. The PD feedback control algorithm, the adaptive feedforward FUVSSLMS control algorithm and the adaptive combined FUVSSLMS control algorithm are implemented for the active vibration control of the thin-walled plate.

\subsection{Experimental identification and filter design}

In order to excite the thin-walled plate structure effectively at its first modal frequency, it is necessary to identify the first modal frequency of the system. In fact, one can use the white noise test, hammering test or swept frequency test to identify the first modal frequency of the system [10]. The swept frequency test is utilized in this paper. A swept frequency sine signal is generated by the signal generator. According to the simulation analysis, the start frequency and the stop frequency are specified as $10 \mathrm{~Hz}$ and $30 \mathrm{~Hz}$, respectively; and the swept time is set as $50 \mathrm{~s}$. After several times of FFT identification, it can be easily known that the first modal frequency of the thin-walled plate is $22.7 \mathrm{~Hz}$.

It is inevitable that some measurement noise and uncorrelated disturbance will be mixed into the measured signal. The noise will seriously weaken the control performance of the experiments. Therefore, the predefined filter for the reference signal and error signal should be designed to process the directly measured vibration signals before conducting the active vibration control experiments. Chebyshev Type I filters are equiripple in the pass band and monotonic in the stop band. Therefore, a Chebyshev Type I filter is designed to filter the vibration signal measured by the reference and error sensor. The experimental results show good filtering performance.

\subsection{Experiments on the active vibration control using the PD feedback control algorithm}

The PD feedback control algorithm is applied as a comparison with the adaptive feedforward and adaptive combined FUVSSLMS control algorithms. Before starting the experiment, it is necessary to define a kind of control performance index when comparing the control performance of different control algorithms. Actually the power spectrum density is widely used for signal analysis and the attenuation of power magnitude can be defined as the control performance index. Since only the first mode vibration of the thin-walled plate is excited and controlled in the experiments, it is only needed to compare the attenuation of power magnitude of the first mode vibration after

conducting different control algorithms. The attenuation of power magnitude can be expressed as

$$
\eta=10 * \log _{10}\left(\frac{p_{1}}{p_{2}}\right) .
$$


where $\eta$ is the attenuation of power magnitude; $p_{1}$ is the power magnitude of the controlled vibration signal with unit $\mathrm{dB}$, and $p_{2}$ is the power magnitude of the uncontrolled vibration signal with unit $\mathrm{dB}$.

Only the error PZT sensor is needed in the PD feedback control experiment. Firstly, the thin-walled plate is excited by the PZT actuators for excitation. Then, the control voltage is applied by the PZT actuators for control at $4.7 \mathrm{~s}$ when the vibration amplitude becomes almost stable. Figure 5 shows the experiment of PD feedback control algorithm with the proportional and derivative control gains of $K_{\mathrm{p}}=3.5, K_{d}=0.015$. From Figure $5(\mathrm{a})$, it can be seen that the excited vibration is suppressed quickly when the control voltage is applied at $4.7 \mathrm{~s}$ and the vibration amplitude attenuates from $1.2 \mathrm{~V}$ to $0.5 \mathrm{~V}$ at $7 \mathrm{~s}$. The control voltage is shown in Figure 5(b). From Figure 5(b), one knows that the vibration amplitude becomes constant after $7 \mathrm{~s}$. The power spectrum density of error signal with and without control is shown in Figure 5(c). From Figure 5(c), it can be seen that the attenuation of power magnitude $\eta$ is $-7.077 \mathrm{~dB}$.

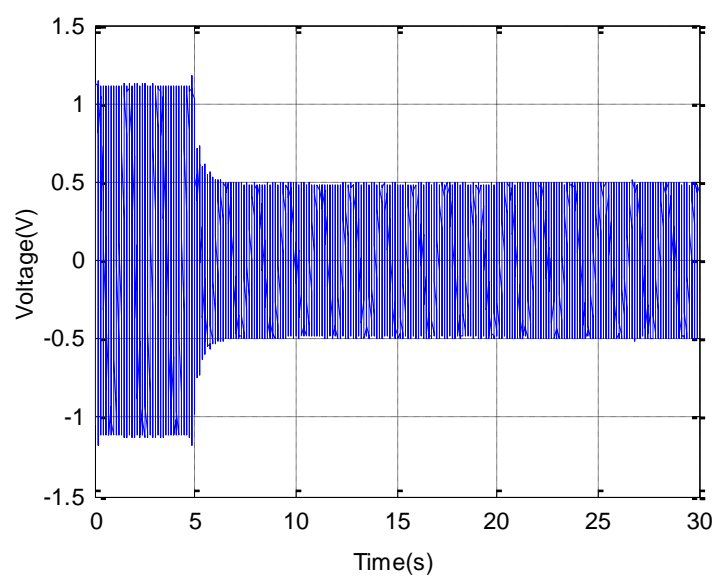

(a) The vibration signal measured by error sensor.

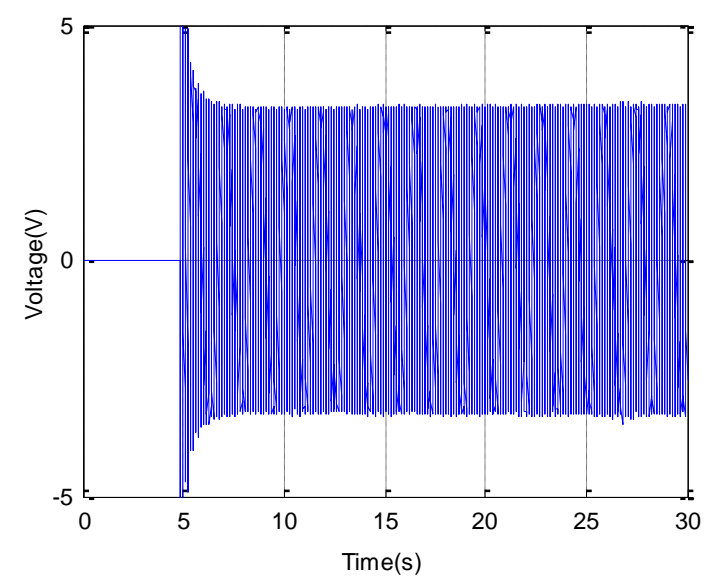

(b) The control voltage.

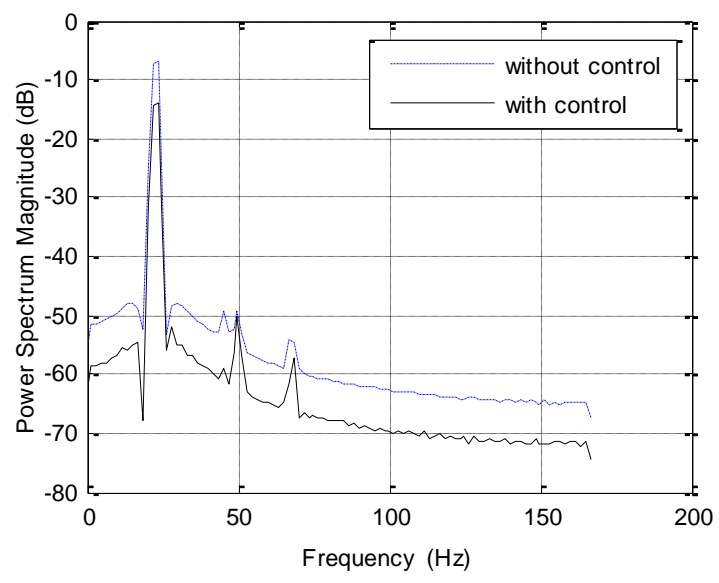

(c) Power spectrum density of error signal with and without control.

Figure 5. The experiment of PD feedback control algorithm with $K_{\mathrm{p}}=3.5, K_{d}=0.015$. 


\subsection{Experiments on vibration control using the adaptive feedforward FUVSSLMS control algorithm}

In order to apply the adaptive feedforward and adaptive combined FUVSSLMS control algorithms, the offline modeling method is used to identify the control path between the PZT actuator for control and the error PZT sensor. The amplitude and frequency of the input sine signal are specified as $1.2 \mathrm{~V}$ and $22.7 \mathrm{~Hz}$, respectively. Generally speaking, when the order of the identified model is larger, the accuracy of the identified model is better. However, it does not mean that the larger order is the better. In practice, an over large order of the identified model will increase the computation burden and make it hard to realize the real-time control. Therefore, it is important to find a proper value of order of the identified control path mode. The order of the identified control path model is chosen as 12 after several tests.

Figure 6 shows the offline control path identification based on the LMS algorithm. The output signal measured by the error PZT sensor and the output signal of the adaptive filter are shown in Figure 6(a) and 6(b), respectively. From Figure 6(b), it can be seen that the output signal of the adaptive filter gradually approaches the output signal measured by the error PZT sensor shown in Figure 6(a), and it remains almost constant after $1.3 \mathrm{~s}$. The error between the measured output and the filter output is shown in Figure 6(c), from which one can see that the error gradually reaches zero after $1.3 \mathrm{~s}$. Then it means that the output of the adaptive filter is almost equal to the output signal measured by the error PZT sensor after 1.3 s. The transfer parameters of the identified control path are illustrated in Figure 6(d) and 6(e). The transfer parameters gradually become constant after $1.3 \mathrm{~s}$. After that, the constant transfer parameters can be used as the estimation of the control path.

At last, the control path model can be expressed as

$$
\begin{aligned}
\boldsymbol{T}_{2}{ }^{\prime}= & -0.0143937493756505-0.00521515815145776 z^{-1}+0.00466769245389309 z^{-2} \\
& +0.0134745650386660 z^{-3}+0.0196169588593735 z^{-4}+0.0219873712630397 z^{-5} \\
& +0.0201586180479408 z^{-6}+0.0144599248879326 z^{-7}+0.00591888784984125 z^{-8} \\
& -0.00392423585880028 z^{-9}-0.0132942429649796 z^{-10}-0.0205022059326921 z^{-11}
\end{aligned} .
$$

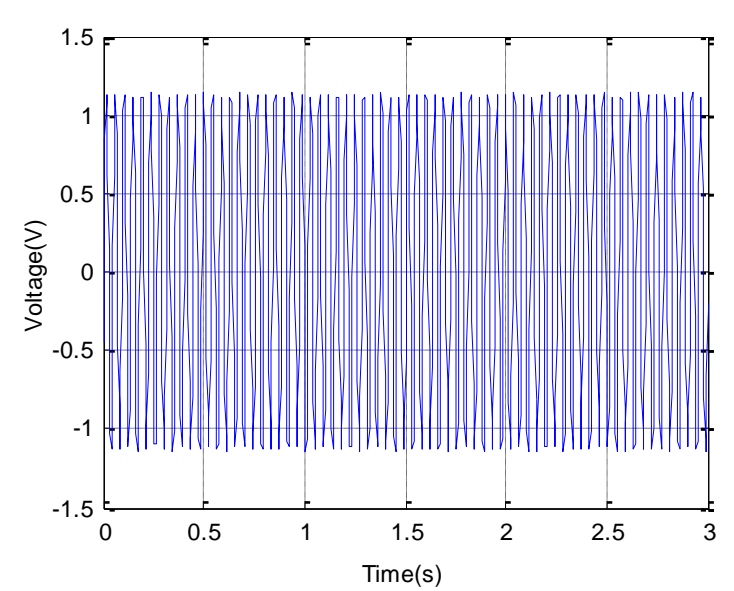

(a) The output signal measured by the error sensor.

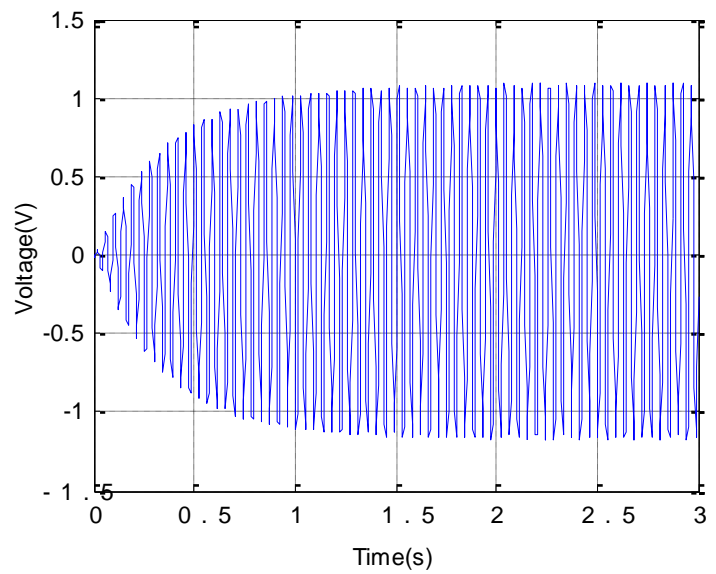

(b) The output signal of the adaptive filter. 


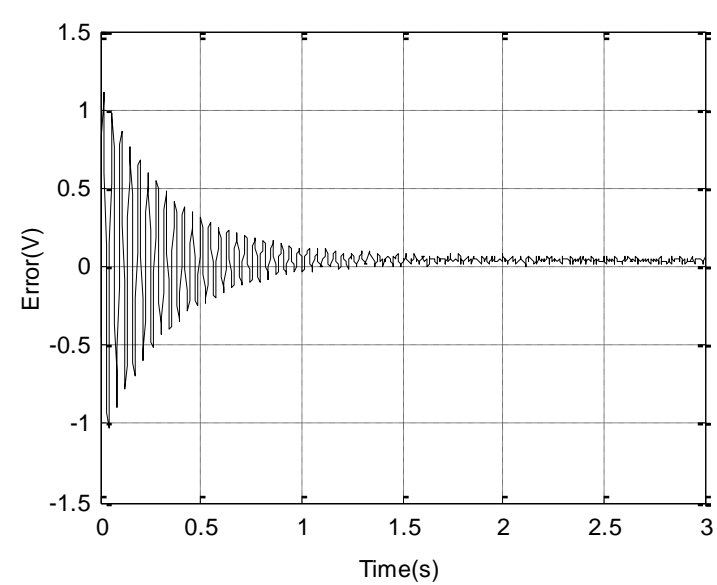

(c) The error between the measured output and the filter output.

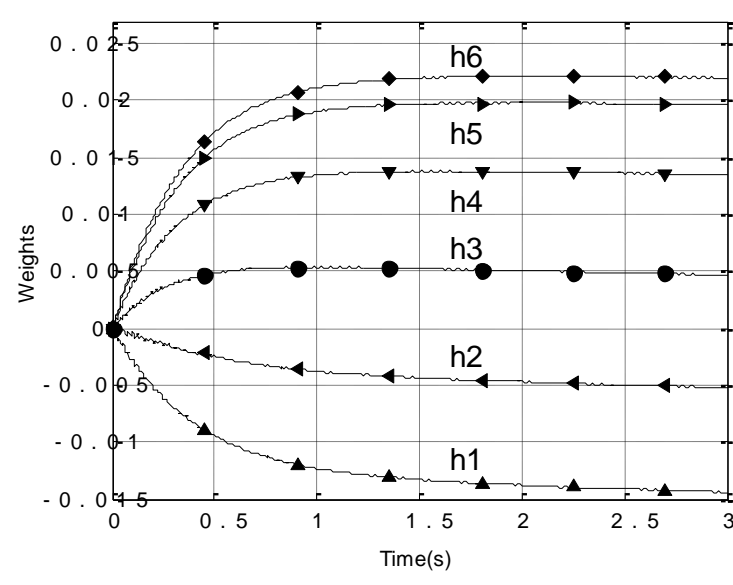

(d) Control path transfer parameters h1-h6.

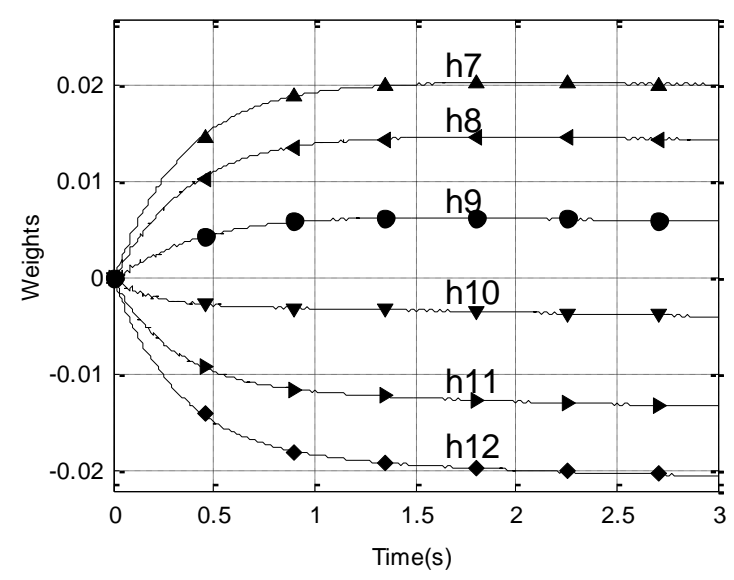

(e) Control path transfer parameters h7-h12.

Figure 6. Experimental identification of the control path.

After obtaining the control path model, the experiment based on the adaptive feedforward FUVSSLMS control algorithm can be conducted. Firstly the convergence factor of the feedback controller $\boldsymbol{W}$ should be set properly to make the control action effective and stable. As mentioned before, the bounds of the convergence factor $\alpha(n)$ is $0<\alpha(n)<1 / \alpha_{\max }$, then $\alpha(n)$ should be set properly by setting the value of parameters $a$ and $b$. After several tests, $a$ is set as 1000 and $b$ is set as 0.005 . Then the convergence factor of the feedback controller $\boldsymbol{V}$ is set as $\beta(n)=0.5^{*} \alpha(n)$. The order of the adaptive filter $\boldsymbol{W}$ and $\boldsymbol{V}$ are set as $O_{1}=6$ and $O_{2}=6$, respectively. The control voltage is applied at $4.7 \mathrm{~s}$ when the vibration voltage reaches the stable amplitude.

The experiments of adaptive feedforward FUVSSLMS control algorithm with $a=1000, b=0.005$, $\beta(n)=0.5^{*} \alpha(n)$ are shown in Figure 7. Figure 7(a) shows the vibration signal measured by the error sensor. The power spectrum density of error signal with and without control is shown in Figure 7(b). From Figure 7(c), one can see 
the vibration signal measured by the reference sensor. In Figure 7(d), the power spectrum density of reference signal with and without control is clearly illustrated. The control voltage is shown in Figure 7(e). Figure 7(f) illustrates the converging process of variable step size $\alpha(n)$. Figure $7(\mathrm{~g})$ and $7(\mathrm{~h})$ demonstrate the converging process of the feedforward controller $\boldsymbol{W}$ weights of a1-a6 and the feedback controller $\boldsymbol{V}$ weights of b1-b6, respectively.
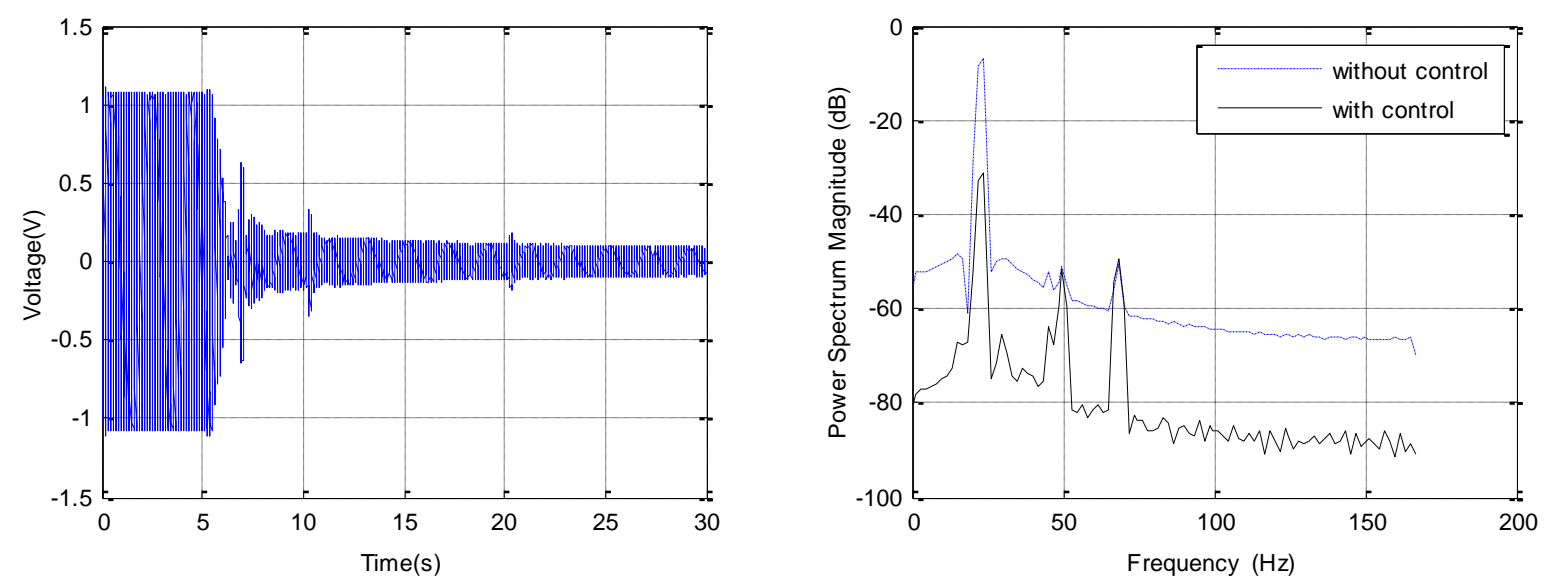

(a) The vibration signal measured by error sensor.

(b) Power spectrum density of error signal with and without control
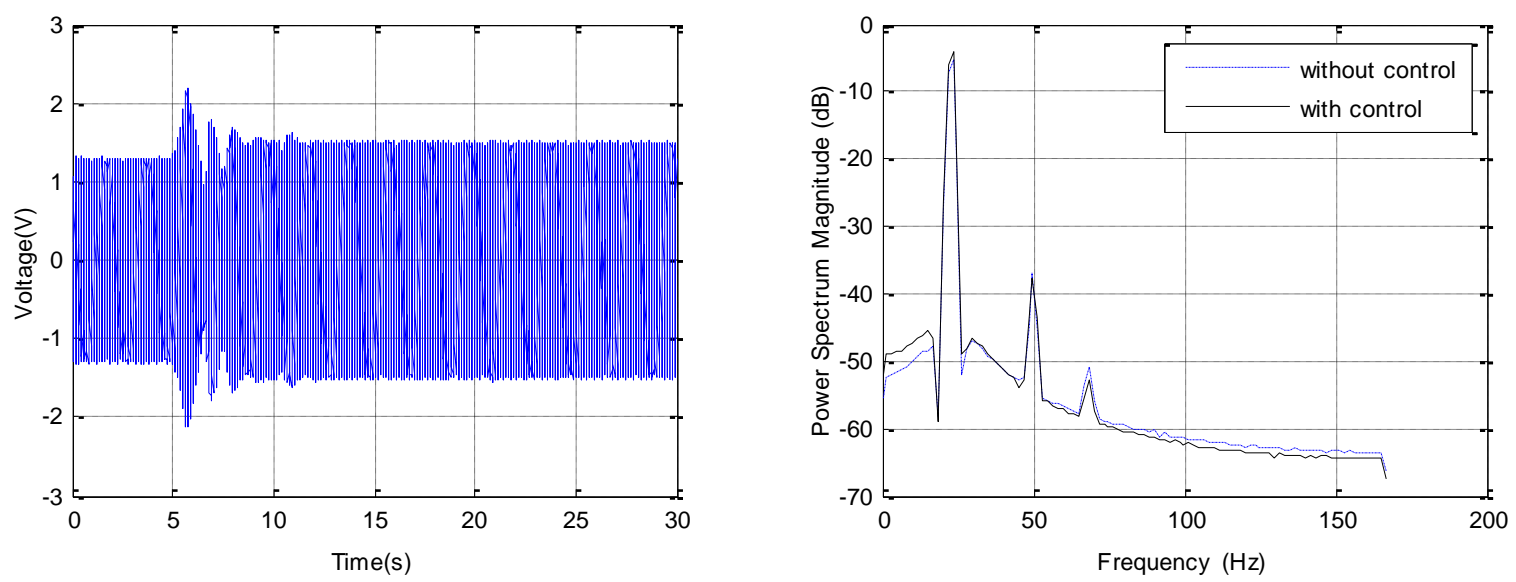

(c) The vibration signal measured by reference sensor. (d) Power spectrum density of reference signal with and without control.

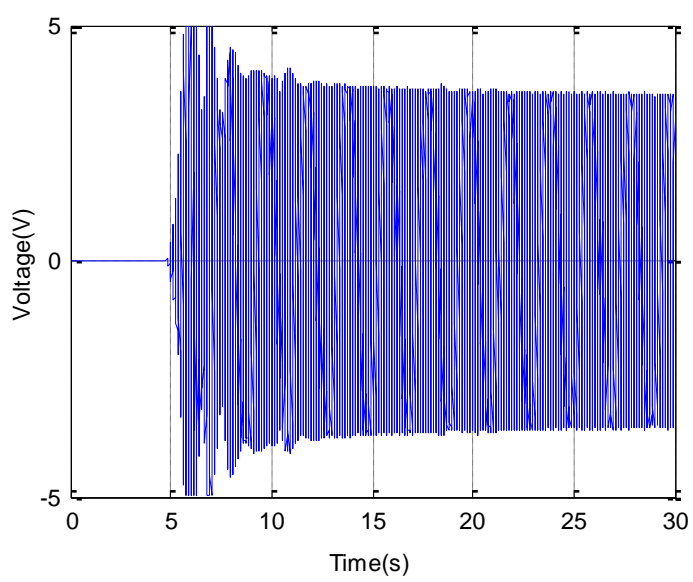

(e) The control voltage.

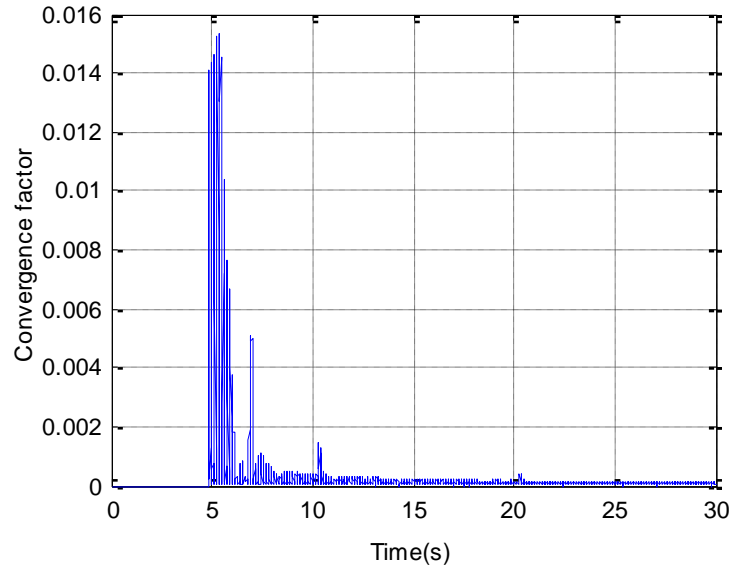

(f) The variable step size $\alpha(n)$. 


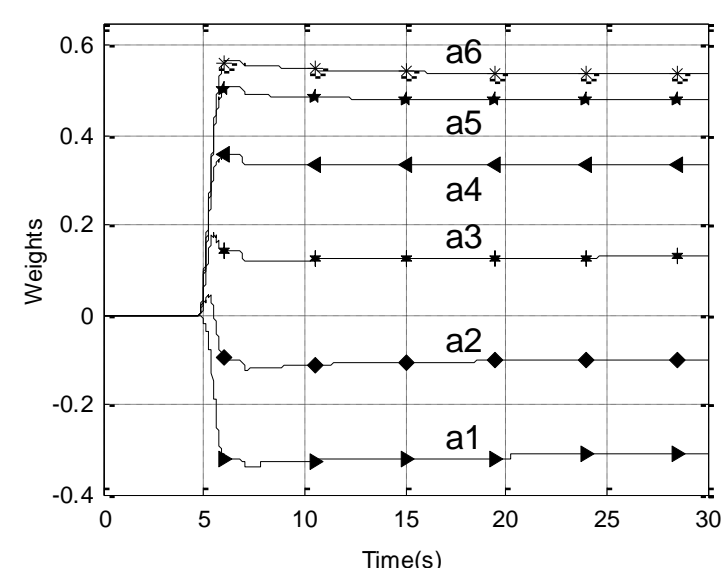

(g) Feedforward controller $\boldsymbol{W}$ weights of a1-a6.

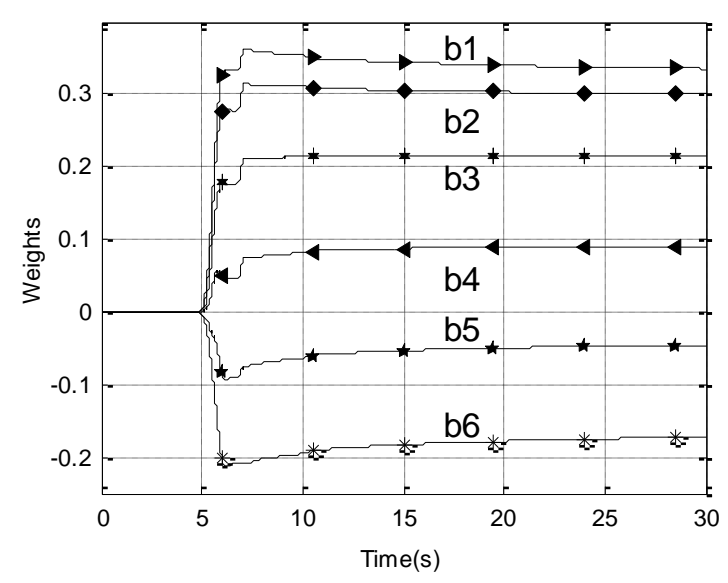

(h) Feedback controller $\boldsymbol{V}$ weights of b1-b6.

Figure 7. The experiment of adaptive feedforward FUVSSLMS control algorithm with $a=1000, b=0.005, \beta(n)=0.5^{*} \alpha(n)$.

In Figure 7(a), it can be seen that once the control voltage is applied at $4.7 \mathrm{~s}$, the vibration of thin-walled plate is suppressed relatively quickly and the vibration amplitude attenuates from $1.2 \mathrm{~V}$ to $0.2 \mathrm{~V}$ after $15 \mathrm{~s}$. Figure $7(\mathrm{~b})$ shows that the attenuation of power magnitude $\eta$ is $-24.259 \mathrm{~dB}$, which demonstrates that the control performance of the adaptive feedforward FUVSSLMS control algorithm is much better than that of the PD feedback control algorithm. From Figure 7(c) and 7(d), one knows that both the amplitude and frequency of reference signals with and without control are almost the same, which means that the reference filter can largely reduce the impact on the reference signal from the positive feedback and measurement noise. From Figure 7(e) it can be seen that in the beginning the control voltage is large enough to reach saturation, then it gradually becomes small and constant value of $3.5 \mathrm{~V}$ after $15 \mathrm{~s}$ as the vibration amplitude attenuates from $1.2 \mathrm{~V}$ to $0.2 \mathrm{~V}$. Also, it can be seen that in the beginning the variable step size $\alpha(n)$ is relatively large, and then it gradually becomes small after the vibration of thin-walled plate has been suppressed in Figure 7(f). In the process of adaptive adjusting, the feedforward and feedback control weights are convergent to constant values quickly as shown in Figure 7(g) and 7(h), respectively.

\subsection{Experiments on the active vibration control using the adaptive combined FUVSSLMS control algorithm}

As previously mentioned, the identified control path model can also be used in the experiment based on the adaptive combined FUVSSLMS control algorithm. In order to carry out a reasonable comparison with the adaptive feedforward FUVSSLMS control algorithm, on one hand, $a$ is also set as 1000 and $b$ is set as 0.005 for the variable step size $\alpha(n)$. On the other hand, the convergence factor of the feedback controller $\boldsymbol{V}$ is also set as $\beta(n)=0.5^{*} \alpha(n)$. The convergence factor of the feedforward controller $\boldsymbol{W}_{1}$ is set as $\gamma(n)=\alpha(n)$, and the convergence factor of the 
feedback controller $\boldsymbol{V}_{1}$ is set as $\varphi(n)=0.5^{*} \alpha(n)$, which are set to be the same as the convergence factors of $\boldsymbol{W}$ and $\boldsymbol{V}$ to keep the control system stable. The orders of the adaptive filter $\boldsymbol{W}, \boldsymbol{V}, \boldsymbol{W}_{1}$ and $\boldsymbol{V}_{1}$ are set as $O_{1}=6$, $O_{2}=6, O_{3}=6$ and $O_{4}=6$, respectively. The control voltage is applied at $4.7 \mathrm{~s}$ when the vibration voltage reaches the stable amplitude.

Figure 8 illustrates the experiment of adaptive combined FUVSSLMS control algorithm with $a=1000$, $b=0.005, \quad \beta(n)=0.5^{*} \alpha(n), \gamma(n)=\alpha(n), \varphi(n)=0.5^{*} \alpha(n)$. Figure $8($ a) shows the vibration signal measured by the error sensor. The power spectrum density of error signal with and without control is illustrated in Figure 8(b). Figure 8(c) demonstrates the vibration signal measured by the reference sensor. In Figure 8(d), one can see the power spectrum density of reference signal with and without control. The control voltage is shown in Figure 8(e) and it is the sum of the control voltage of the feedforward controller in Figure 8(f) and the control voltage of the feedback controller in Figure $8(\mathrm{~g})$. The converging process of variable step size $\alpha(n)$ is shown in Figure $8(\mathrm{~h})$. Figure 8(i) and 8(j) illustrate the converging process of the feedforward controller $\boldsymbol{W}$ weights of a1-a6 and the feedback controller $\boldsymbol{V}$ weights of b1-b6, respectively. Figure $8(\mathrm{k})$ and $8(\mathrm{l})$ show the converging process of the feedforward controller $\boldsymbol{W}_{1}$ weights of c1-c6 and the feedback controller $V_{1}$ weights of d1-d6, respectively.

From Figure 8(a) it can be seen that once the control voltage is applied at $4.7 \mathrm{~s}$. The vibration of vibration plate is suppressed more quickly than that of the adaptive feedforward FUVSSLMS control algorithm as shown in Figure 7(a). It also can be seen that the vibration amplitude attenuates from $1.2 \mathrm{~V}$ to $0.1 \mathrm{~V}$ after $10 \mathrm{~s}$. Figure $8(\mathrm{~b})$ shows that the attenuation of power magnitude $\eta$ is $-33.546 \mathrm{~dB}$, which demonstrates that the control performance of the adaptive combined FUVSSLMS control algorithm is much better than that of the adaptive feedforward FUVSSLMS control algorithm as shown in Figure 7(b). In Figure 8(c) and 8(d), one knows that both the amplitude and frequency of reference signals with and without control are almost the same, which shows that the reference filter can largely reduce the impact on the reference signal from the positive feedback and measurement noise. From Figure $8(\mathrm{e})$ one can see that in the beginning the control voltage is large enough to reach saturation just as that shown in Figure 7(e), but it can quickly become small and constant value of $3.7 \mathrm{~V}$ quickly after $10 \mathrm{~s}$ as the vibration amplitude attenuates from $1.2 \mathrm{~V}$ to $0.1 \mathrm{~V}$. This demonstrates that by using almost the same control voltage, the control performance of the adaptive combined FUVSSLMS control algorithm is much better than that of the adaptive feedforward FUVSSLMS control algorithm. From Figure 8(f) and 8(g), one can also know that the control voltage of the feedback controller is just a little smaller than the control voltage of the feedforward controller, which shows that the feedback controller also play an important role in the adaptive combined FUVSSLMS control algorithm. It also can be seen that the variable step size 
$\alpha(n)$ in Figure 8(h) converges faster than that in Figure 7(f). In the process of adaptive adjusting, the feedforward and feedback control weights are also convergent to constant values quickly as shown in Figure 8(i), 8(j), 8(k) and 8(l), respectively.

The control performance of the given control algorithms is shown in Table 3. From Table 3 one knows that on one hand, the adaptive combined FUVSSLMS control algorithm has the largest attenuation of power magnitude with $-33.546 \mathrm{~dB}$ and the PD feedback control algorithm has the shortest convergence time with $2.3 \mathrm{~s}$. On the other hand, the adaptive combined FUVSSLMS control algorithm has both a larger attenuation of power magnitude and a shorter convergence time than the adaptive feedforward FUVSSLMS control algorithm. In conclusion, we can say that the adaptive combined FUVSSLMS control algorithm has better control performance than the other two control algorithms for a relatively long-time experiment.

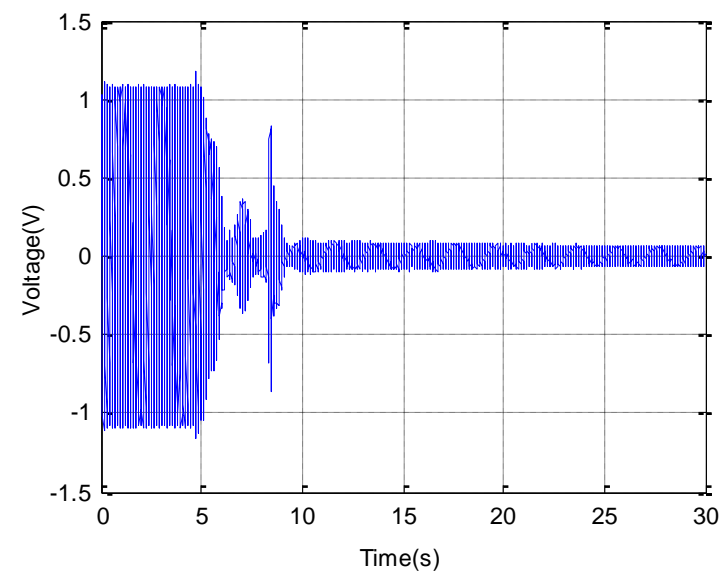

(a) The vibration signal measured by error sensor.

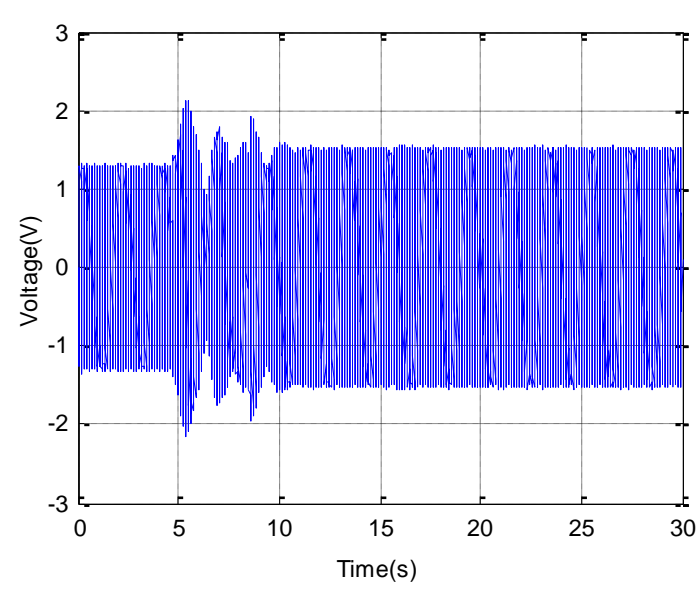

(c) The vibration signal measured by reference sensor.

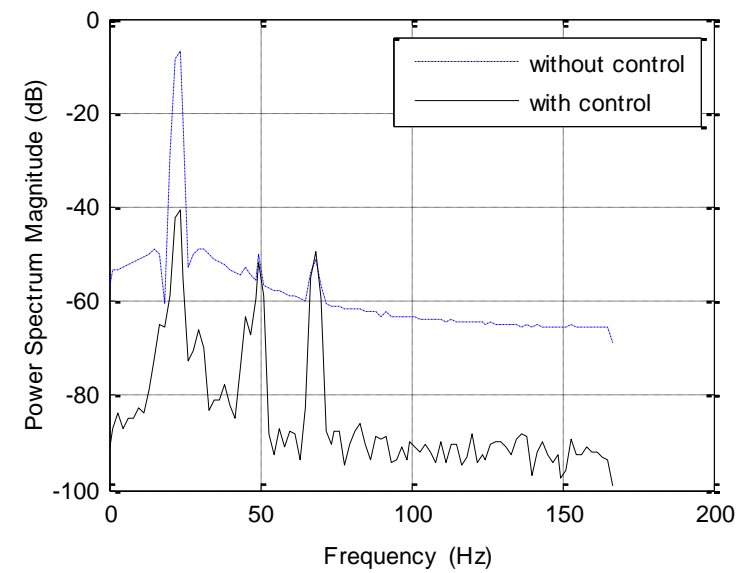

(b) Power spectrum density of error signal with and without control.

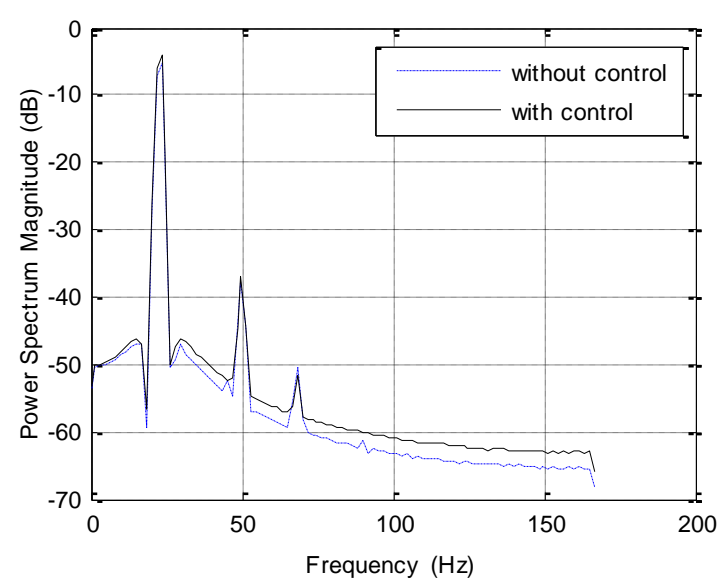

(d) Power spectrum density of reference signal with and without control. 


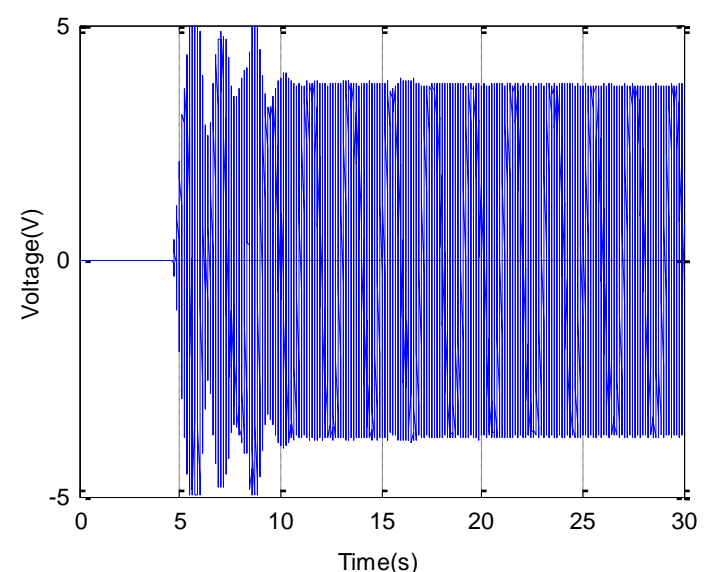

(e) The control voltage.

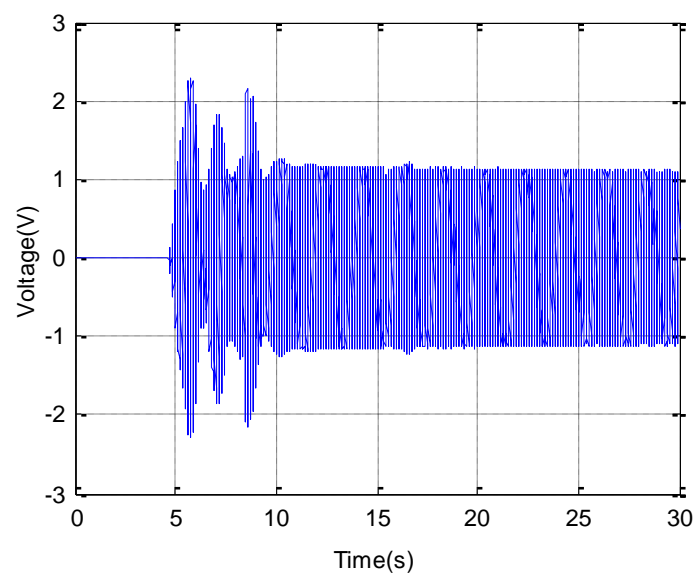

(g) Control voltage of the feedback controller.

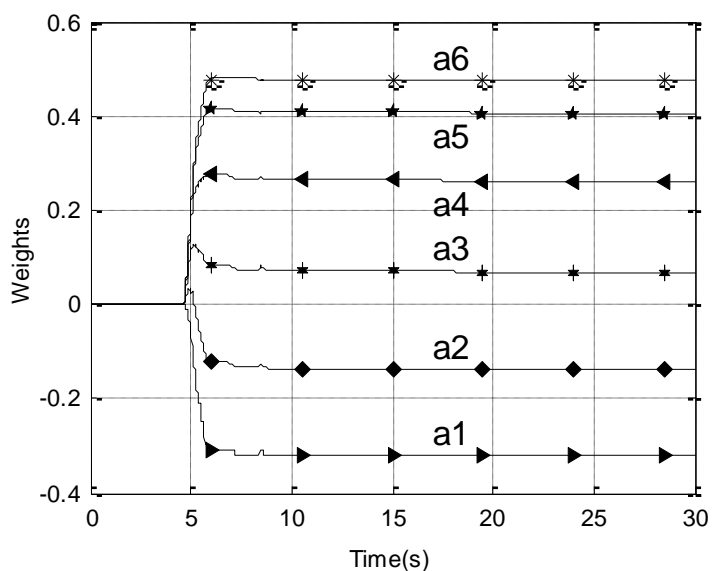

(i) Feedforward controller $\boldsymbol{W}$ weights of a1-a6.

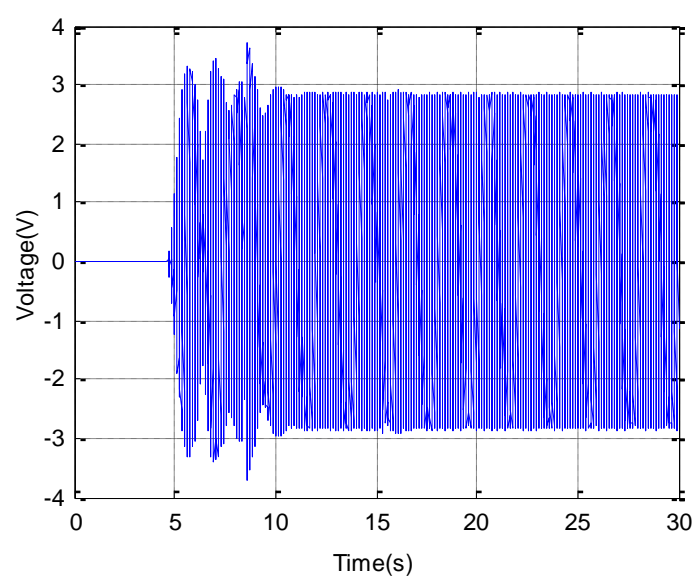

(f) Control voltage of the feedforward controller.

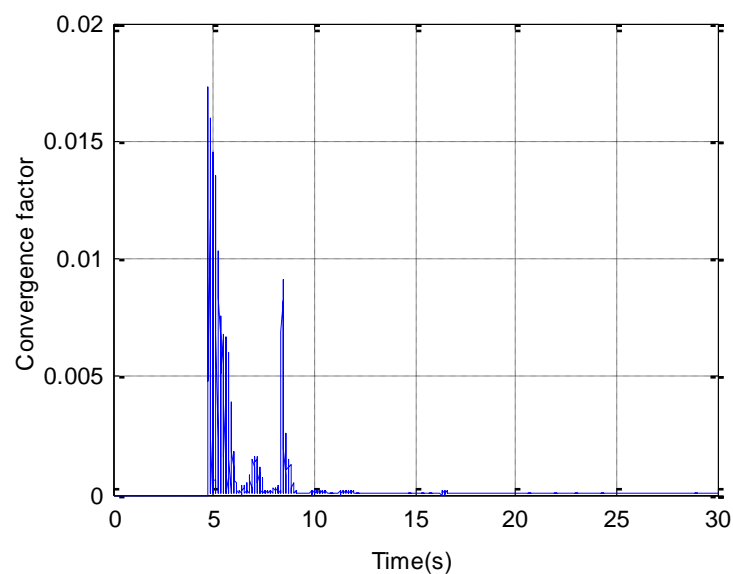

(h) The variable step size $\alpha(n)$.

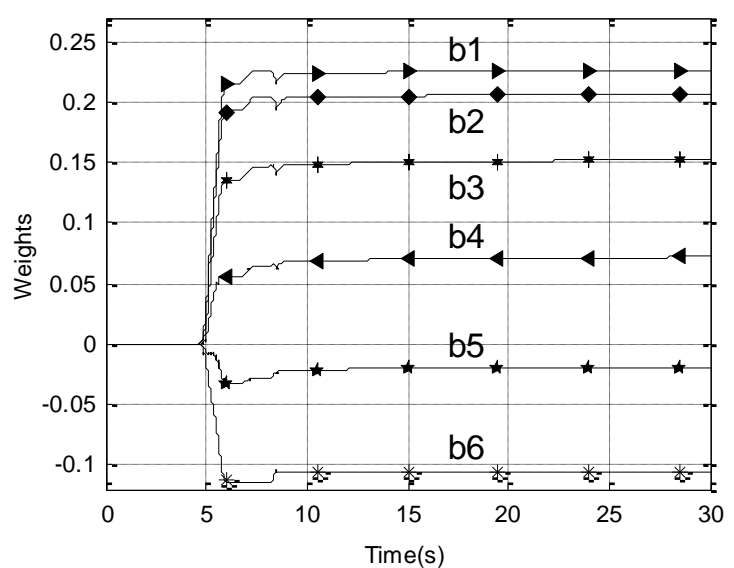

(j) Feedback controller $\boldsymbol{V}$ weights of b1-b6. 


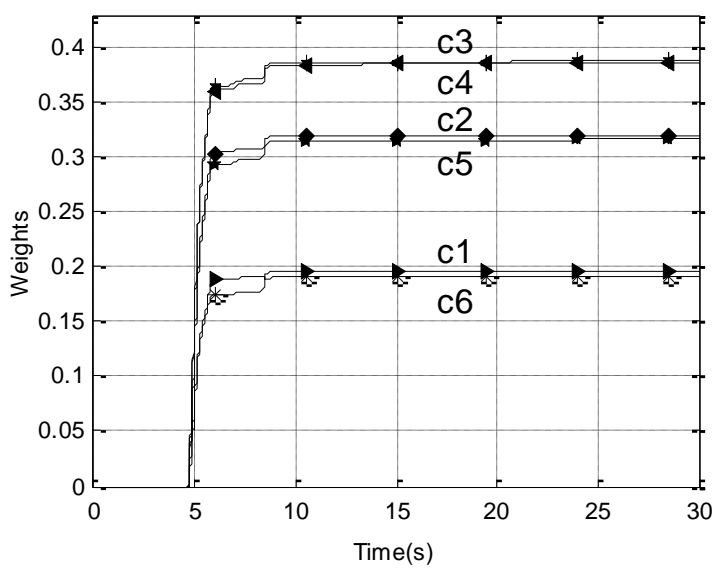

(k) Feedforward controller $\boldsymbol{W}_{1}$ weights of c1-c6.

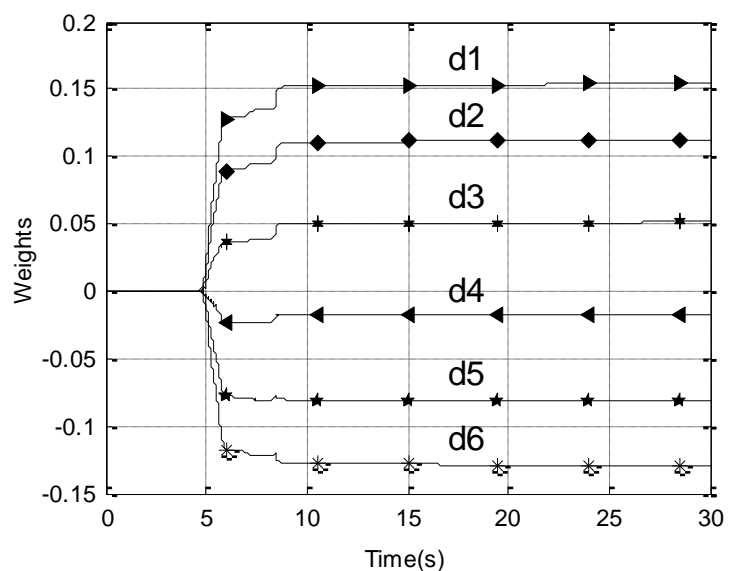

(1) Feedback controller $\boldsymbol{V}_{1}$ weights of d1-d6.

Figure 8. The experiment of adaptive combined FUVSSLMS control algorithm with

$$
a=1000, b=0.005, \beta(n)=0.5^{*} \alpha(n), \gamma(n)=\alpha(n), \varphi(n)=0.5^{*} \alpha(n) .
$$

Table 3. The control performance of the given control algorithms.

\begin{tabular}{ccc}
\hline Control algorithm & The attenuation of power magnitude (dB) & Convergence time (s) \\
\hline The PD feedback control & -7.077 & 2.3 \\
The adaptive feedforward FUVSSLMS control & -24.259 & 10.3 \\
The adaptive combined FUVSSLMS control & -33.546 & 5.3 \\
\hline
\end{tabular}

\section{Conclusions}

This paper presents a kind of adaptive feedforward and combined FUVSSLMS vibration control system with variable step size and reference filter to control the vibration of thin-walled plate. Firstly, the theoretical analysis of adaptive feedforward and combined FUVSSLMS control algorithms with variable step size and reference filter is carried out. Secondly, a set of active vibration control experimental setup with PZT sensors and PZT actuators is constructed. Thirdly, the experiments of the PD feedback control algorithm and the adaptive feedforward FUVSSLMS control algorithm are applied for comparisons. Finally, the experiment of the adaptive combined FUVSSLMS control algorithm is conducted. The experimental results demonstrate that the adaptive combined FUVSSLMS control algorithm has better control performance than the other two control algorithms. In the experiments, the variable step size can result in fast convergence speed and low residual error. The reference filter is used to extract the desired signal from the positive feedback and measurement noise and the filtering results show good performance. 


\section{Acknowledgements}

The authors gratefully acknowledge these support agencies such as the National Science Foundation of China (Grant No. 51175181), the Fundamental Research Funds for the Central Universities, SCUT, and the Natural Science Foundation of Guangdong Province (S2013030013355).

\section{References}

[1] Wu SY, Turner TL and Rizzi SA. Piezoelectric shunt vibration damping of an F-15 panel under high-acoustic excitation, SPIE's 7th Annual International Symposium on Smart Structures and Materials, International Society for Optics and Photonics 276-287 (2000).

[2] Zhi-cheng Qiu, Jian-da Han, Xian-min Zhang, Yue-chao Wang, Zhen-wei Wu. Active vibration control of a flexible beam using a non-collocated acceleration sensor and piezoelectric patch actuator. Journal of Sound and Vibration 326 (2009) $438-455$.

[3] Osama Abdeljaber, Onur Avci, Daniel J. Inman. Active vibration control of flexible cantilever plates using piezoelectric materials and artificial neural networks, Journal of Sound and Vibration, 363 (2016) 33-53.

[4] I.S. Sadek, I. Kucuk, S. Adali, Active open-loop control of plates with multiple piezoelectric patches via the maximum principle, Mechanics of Advanced Materials and Structures 21 (2014) 772- 779

[5] Jinhao Qiu and Masakazu Haraguchi. Vibration control of a plate using a self-sensing piezoelectric actuator and an adaptive control approach, Journal of Intelligent Material Systems And Structures, 17 (2006) 661-669.

[6] Eriksson L. (1991) Development of the filtered-U algorithm for active noise control. The Journal of the Acoustical Society of America 89: 257-265.

[7] Ho-Wuk Kim, Hong-Sug Park, Sang-Kwon Lee and Kihong Shin. Modified-filtered-u LMS algorithm for active noise control and its application to a short acoustic duct. Mechanical Systems and Signal Processing 25 (2011) 475-484.

[8] J. H. PARK and S. K. LEE. A novel adaptive algorithm with an IIR filter and a variable step size for active noise control in a short duct. International Journal of Automotive Technology, Vol. 13, No. 2, pp. 223-229 (2012).

[9] Zhiyuan Gao, Xiaojin Zhu, Quanzhen Huang, et al. Andysis and implementation of FULMS algorithm based active vibration control system[J]. Communications in Computer and Information Science 2010, Vol.97, Part 1, PP: 56-63.

[10] Lingbo Xie, Zhi-cheng Qiu and Xian-min Zhang. Vibration control of a flexible clamped-clamped plate based on an improved FULMS algorithm and laser displacement measurement. Mechanical Systems and Signal Processing 75 (2016) $209-227$.

[11] Khaled Mayyas. A variable step-size selective partial update LMS algorithm. Digital Signal Processing 23 (2013) 75-85.

[12] Landau I D, Airimitoaie T B and Alma M. A Youla-Kucera parametrized adaptive feedforward compensator for active vibration control with mechanical coupling. Automatica, 2012, 48(9): 2152-2158.

[13] S. Nima Mahmoodi, Mehdi Ahmadian, and Daniel J. Inman. Adaptive modified positive position feedback for active vibration control of structures, Journal of Intelligent Material Systems and Structures, 21 (2010) 571-580.

[14] Alexander D. Streeter, Laura R. Ray, and Robert D. Collier. Hybrid Feedforward-Feedback Active Noise Control. Proceeding of the 2004 American Control Conference, Boston, Massachusetts June 30 - July 2, 2004. 
[15] Gao Zhiyuan, Zhu Xiaojin, Zhang Hesheng, Luo Cong and Li Mingdong. Analysis and implementation of FURLS algorithm for active vibration control system with positive feedback. HIGH TECHNOLOGY LETTERS, Vol.21 No.2, June 2015, pp.171-177.

[16] Ioan Dor'e Landau and Tudor-Bogdan Airimitoaie. A General Adaptive Feedforward Compensation Algorithm for Active Vibration Control with Mechanical Coupling and Local Feedback. 2014 22nd Mediterranean Conference on Control and Automation (MED) University of Palermo. June 16-19, 2014. Palermo, Italy.

[17] GAO Zhi-yuan, ZHU Xiao-jin, ZHANG He-sheng, LI Pei-jiang. Hybrid adaptive control method for active vibration control system with positive feedback. Control Theory \& Applications Vol. 32 No. 7 Jul. 2015.

[18] Shouda Jiang, Zhong Bo, Chao Sun, and Yuqi Liu. A Hybrid Narrowband Active Noise Control System for Uncorrelated Narrowband Disturbance. Journal of Information Hiding and Multimedia Signal Processing Volume6, Number 2, March 2015.

[19] S.R. Chen, G.-P.J. Too. Simulations and experiments for hybrid noise control systems. Applied Acoustics 70 (2009) $247-255$.

[20] Biao Ma, Zhi-cheng Qiu, Xian-min Zhang, Jian-da Han. Experiments on resonant vibration suppression of a piezoelectric flexible clamped-clamped plate using filtered-U least mean square algorithm, Journal of Intelligent Material Systems and Structures.27 (2016) $166-194$.

[21] Quanzhen Huang, Xiaojin Zhu, Zhiyuan Gao, Shouwei Gao and Enyu Jiang. Analysis and implementation of improved multi-input multi-out put filtered-X least mean square algorithm for active structural vibration control. Structural Control and Health Monitoring. 2013; 20:1351- 1365.

[22] LU Bing-qian, FENG Cun-qian and LONG Ge-nong. A new variable step-size LMS algorithm based on sine function. Journal of Air Force Engineering University(Natural Science Edition) 14:47-51 (2013).

[23] Zhi-cheng Qiu and Biao Ma. Adaptive resonant vibration control of a piezoelectric flexible plate implementing filtered-X LMS algorithm. International Journal of Acoustics and Vibration 19:224 -239 (2013). 